\title{
Postoperative ileus in an enhanced recovery pathway-a retrospective cohort study
}

\author{
Fabian Grass $^{1}$ • Juliette Slieker ${ }^{1}$ - Jonas Jurt ${ }^{1}$ - Anne Kummer ${ }^{1}$ - Josep Solà ${ }^{2}$. \\ Dieter Hahnloser $^{1}$ • Nicolas Demartines ${ }^{1}$ • Martin Hübner ${ }^{1}$
}

Accepted: 3 March 2017 / Published online: 11 March 2017

(C) Springer-Verlag Berlin Heidelberg 2017

\begin{abstract}
Purpose Enhanced recovery after surgery (ERAS) protocols advocate no nasogastric tubes after colorectal surgery, but postoperative ileus (POI) remains a challenging clinical reality. The aim of this study was to assess incidence and risk factors of POI.

Methods This retrospective analysis included all consecutive colorectal surgical procedures since May 2011 until November 2014. Uni- and multivariate risk factors for POI were identified by multiple logistic regression and functional and surgical outcomes assessed.

Results The study cohort consisted of 513 consecutive colorectal ERAS patients. One hundred twenty-eight patients (24.7\%) needed postoperative reinsertion of nasogastric tube at the $3.9 \pm 2.9$ postoperative day. Multivariate analysis retained the American Society of Anesthesiologists group 3-4 (odds ratio (OR) $1.3 ; 95 \%$ CI $1-1.8, p=0.043$ ) and duration of surgery of $>3 \mathrm{~h}(\mathrm{OR} 1.3 ; 95 \% \mathrm{CI} 1-1.7, p=0.047)$ as independent risk factors for POI. Minimally invasive surgery (OR $0.6 ; 95 \% \mathrm{CI}$ $0.5-0.8, p \leq 0.001)$ and overall compliance of $>70 \%$ to the ERAS protocol (OR 0.7; 95\% CI 0.6-1, $p=0.031$ ) represented independent protective factors. POI was associated with respiratory ( 23 vs. $5 \%, p \leq 0.001)$ and cardiovascular (16 vs. $3 \%$, $p \leq 0.001)$ complications.
\end{abstract}

This study was presented at the Annual Swiss Surgical Meeting 2015, May 20-22, Bern, Switzerland.

Nicolas Demartines

demartines@chuv.ch

1 Department of Visceral Surgery, University Hospital of Lausanne (CHUV), Bugnon 46, 1011 Lausanne, Switzerland

2 Centre Suisse d'Electronique et de Microtechnique (CSEM), Neuchâtel, Switzerland
Conclusions POI was frequent in the present study. Overall compliance to the ERAS protocol and minimally invasive surgery helped to prevent POI, which was significantly correlated with medical complications.

Keywords Enhanced recovery $\cdot$ Fast track $\cdot$ Colorectal . Ileus $\cdot$ Nasogastric drainage $\cdot$ Compliance

\section{Introduction}

Postoperative ileus (POI) remains a challenging clinical reality affecting up to $25 \%$ of the patients [1]. In a recent analysis, prolonged ileus was the postoperative complication associated with the largest increase in length of stay [2]. Several items of the enhanced recovery after surgery (ERAS) protocol target POI. In particular, the administration of systematic laxatives, stringent fluid management and avoiding of opioids or prophylactic nasogastric drains have demonstrated their efficacy in the reduction of POI [3].

The aim of the present study was to assess the incidence of postoperative ileus in patients treated within a comprehensive ERAS pathway. Further, risk factors for POI and outcome of these patients were analysed.

\section{Methods}

This retrospective study was conducted by analysis of a prospective database of consecutive full age patients who underwent colorectal surgical procedures within the ERAS pathway in the University Hospital of Lausanne between May 2011 and November 2014. All patients undergoing major open or laparoscopic colorectal surgery including related stoma procedures in an elective or emergency (since April 2012) 
setting were included in the ERAS protocol. This study was approved by the Institutional Review Board (Commission cantonale d'éthique de la recherche sur l'être humain (CERVD)), and informed consent was obtained from every patient. Patient records and information were anonymized and deidentified prior to analysis. The study was conducted according to the STROBE criteria and registered under www. researchregistry.com (UIN research registry 1034).

Pertinent demographic and surgical information was prospectively assessed in a dedicated database by two dedicated ERAS nurses; accuracy of data entry was cross-checked by independent review. Demographic information included age, gender, body mass index (BMI), American Society of Anesthesiologists (ASA) and World Health Organization (WHO) mobility performance scores, social habits such as smoking or alcohol abuse (use of alcoholic beverages to excess as a regular practice) at the time of the procedure and comorbidities, i.e. cardiac history (including congestive heart failure, ischemic and dysrhythmic conditions) and compromising respiratory disease, diabetes mellitus or previous surgery to the same abdominal region. Further, previous episodes of postoperative nausea or vomiting (PONV) during a former procedure, the presence of a malignant indication for the present surgery and prior radiotherapy to the operating field, including neoadjuvant radiotherapy, were recorded. Surgical information included main procedure (left, right or total colectomy; rectal resection; stoma procedure, in particular Hartmann reversal, loop ileostomy closure or colostomy realization and closure and other), surgical approach (minimally invasive or open), duration, setting (elective or emergency, defined as any procedure performed during an unplanned hospital admission) and magnitude (major or extended major surgery) of the surgical procedure. Extended major surgery (low rectum resection, proctocolectomy, total colectomy and ileal pouch-anal anastomosis) was distinguished from major surgery (segmental colectomy and Hartmann reversal). Further, the realization of a new stoma, either protective or end stoma, and the realization of an anastomosis were recorded.

All perioperative care items of the ERAS protocol [3] were systematically recorded in the ERAS Interactive Audit System. Overall compliance to 19 pre-, peri- and postoperative ERAS care items was assessed and stratified with a cutoff of 70\% according to Gustafsson et al. [4] and our own institutional experience (unpublished data). These items were preadmission patient education, no oral bowel preparation, preoperative oral carbohydrate drinks, no preoperative long acting sedative medication, thrombo-prophylaxis, antibiotic prophylaxis, PONV prophylaxis (droperidol $1 \mathrm{mg}$, ondansetron $4 \mathrm{mg}$, bethamethasone $4 \mathrm{mg}$ ), intraoperative thoracic epidural analgesia, hypothermia prevention (active warming by air blanket), fluid administration guidance, balanced intravenous fluids $(\mathrm{m} / \mathrm{kg} / \mathrm{h}<7)$, no prophylactic nasogastric tube (NGT), no abdominal drain, sip feeds at postoperative day (POD) 0 of $>300 \mathrm{kcal}$, sufficient oral fluids (>1 L) at POD 0 , systematic laxatives, IV fluid administration at POD 1 of $<500 \mathrm{~mL}$, weight gain of $<1.5 \mathrm{~kg}$ at POD 1 and mobilization at all at POD 0.

Visual analogue scales (VAS 1-10) were used to assess postoperative pain and nausea. Clinical outcome was evaluated until 30 days postoperatively. Medical complications were assessed, in particular, respiratory failure or pneumonia, cardiovascular complications, including dysrhythmia and angina pectoris or myocardial infarction and urinary tract infections. Major complications (Clavien III+) were classified according to the Clavien classification score [5]. Further, length of stay and readmission rates were recorded.

\section{Outcomes/study endpoints}

POI was the primary endpoint, and it was defined as the need for reinsertion of a NGT. Data about timing, duration, second reinsertion and whether a Gastrografin challenge (administration of $100 \mathrm{~mL}$ of Gastrografin and clamping of the NGT for $4 \mathrm{~h}$, to stimulate gastrointestinal activity) was performed were recorded. Risk factors for POI were identified among demographic, surgical and ERAS-related parameters. In a second step, medical (respiratory, cardiovascular and urinary) and major complications, length of stay and readmission rates were compared between the two groups (POI vs. no POI).

\section{Statistical analysis}

Descriptive statistics for categorical variables were reported as frequency (\%), while continuous variables were reported as mean (standard deviation) or median (interquartile range). Chi-square was used for comparison of categorical variables. All statistical tests were two sided, and a level of 0.05 was used to indicate statistical significance. Variables with $p$ values $\leq 0.05$ were then entered into a multivariate logistic regression (based on a probit regression model) to provide adjusted estimations of the odds ratio (OR). Data analysis was performed with the Statistical Software for the Social Sciences (SPSS) Advanced Statistics 22 (IBM Software Group, 200 W. Madison St., Chicago, IL; 60606, USA) and MATLAB Statistical Toolbox v10.1 (MathWorks, 1 Apple Hill Drive, Natick, MA; 01760-2098, USA).

\section{Results}

\section{Patients}

A total of 513 patients (276 males and 237 females) with a mean age of $63 \pm 16$ years underwent colorectal surgical 
procedures during the study period. Table 1 gives an overview of baseline demographic characteristics.

Left colectomies and sigmoid resections were performed in 144 patients (28\%), right colectomies and ileocaecal resections in 106 patients (21\%) and total or subtotal colectomies and proctocolectomies in 29 patients $(6 \%)$. Rectum procedures, mainly low anterior resections, were performed in 123 patients (24\%). Stoma procedures, mainly Hartmann reversals and loop ileostomy closures, were performed in 84 patients $(16 \%)$.

Surgical details are displayed in Table 2. In the emergency setting, $30 \%$ of the colorectal resections were performed by a minimally invasive approach, in contrast to $75 \%$ in the elective setting.

\section{Incidence of POI}

One hundred and twenty-eight patients $(24.7 \%)$ presented with POI. NGTs were reinserted at POD 1 in 6 patients (5\%), POD 2 in 31 patients (24\%), POD 3 in 38 patients $(30 \%)$ and POD 4 or later in 53 patients (41\%). POI occurred after a mean time lapse of $3.9 \pm 2.9$ days, and NGTs were maintained for a mean time of $54 \pm 52 \mathrm{~h}$. Thirty-four patients (7\%) had a prophylactic postoperative nasogastric tube. A second NGT was necessary in 25 patients $(20 \%)$, and Gastrografin challenge was performed in 41 patients $(32 \%)$.

Table 1 Demographics

\begin{tabular}{llll}
\hline & POI $(n=128)$ & No POI $(n=385)$ & $p$ \\
\hline Age & $64 \pm 14$ & $63 \pm 17$ & 0.322 \\
Gender $(\mathrm{m} / \mathrm{f})$ & $72: 56$ & $204: 181$ & 0.521 \\
BMI $\left(\mathrm{kg} / \mathrm{m}^{2}\right)$ & $25.4 \pm 4.8$ & $25.4 \pm 4.6$ & 0.978 \\
ASA group $(1-2: 3-4)$ & $80: 48$ & $297: 87$ & 0.001 \\
Smoking & $24 / 128(19 \%)$ & $79 / 384(21 \%)$ & 0.656 \\
Alcohol abuse & $12 / 108(11 \%)$ & $26 / 336(8 \%)$ & 0.441 \\
Diabetes Mellitus & $14 / 128(11 \%)$ & $42 / 384(10.9 \%)$ & 1 \\
Previous PONV & $11 / 121(9 \%)$ & $36 / 373(10 \%)$ & 0.855 \\
WHO score $>2$ & $20 / 128(16 \%)$ & $25 / 381(7 \%)$ & 0.002 \\
Prior radiotherapy & $19 / 128(15 \%)$ & $44 / 384(12 \%)$ & 0.313 \\
Previous abdominal surgery & $68 / 128(53 \%)$ & $168 / 384(44 \%)$ & 0.065 \\
Cardiac history & $52 / 128(41 \%)$ & $139 / 385(36 \%)$ & 0.359 \\
Respiratory history & $18 / 127(14 \%)$ & $52 / 385(14 \%)$ & 0.85 \\
Malignant indication & $61 / 128(48 \%)$ & $171 / 383(45 \%)$ & 0.554 \\
\hline
\end{tabular}

Baseline demographic parameters of patients with postoperative ileus $(n=128)$ and patients without postoperative ileus $(n=385)$. Age and $\mathrm{BMI}$ are mean \pm standard deviation. All others are frequency with percentages. Italicized characters indicate significant values $(p<0.05)$

$P O I$ postoperative ileus, $B M I$ body mass index, $A S A$ American Society of Anesthesiologists, $P O N V$ postoperative nausea and vomiting, $W H O$ World Health Organization
Table 2 Surgical parameter

\begin{tabular}{llll}
\hline & POI $(n=128)$ & No POI $(n=385)$ & $p$ \\
\hline Minimally invasive surgery & $42(33 \%)$ & $204(53 \%)$ & $<0.001$ \\
Emergency indication & $35(27 \%)$ & $65(17 \%)$ & 0.01 \\
Extended major surgery & $31(24 \%)$ & $66(17 \%)$ & 0.077 \\
Duration $>3$ h & $80(63 \%)$ & $186(48 \%)$ & 0.005 \\
New stoma & $47(37 \%)$ & $88(23 \%)$ & 0.002 \\
Anastomosis & $107(84 \%)$ & $333(86 \%)$ & 0.416 \\
\hline
\end{tabular}

Surgical details of patients with postoperative ileus $(n=128)$ and patients without postoperative ileus $(n=385)$. Italicized characters indicate significant values $(p<0.05)$

$P O I$ postoperative ileus

\section{Univariate and multivariate risk factors for POI}

Patients presenting with POI had higher ASA scores (ASA group 3-4: 38 vs. $23 \%, p=0.001$ ) and higher WHO mobility performance scores (WHO score $>2: 16$ vs. $7 \%, p=0.002$ ) (Table 1).

Among surgery-related items, duration of the operation $(220 \pm 100$ vs. $190 \pm 90 \mathrm{~min}, p=0.001)$, emergency indication ( 27 vs. $17 \%, p=0.001$ ) and realization of a new stoma ( 37 vs. $23 \%, p=0.002)$ were univariate risk factors for POI, whereas minimally invasive surgery was a protective factor (33 vs. $53 \%, p \leq 0.001$ ) (Table 2).

ERAS-related items are displayed in Fig. 1. Use of abdominal drains ( 29 vs. $20 \%, p=0.036$ ), urinary catheters (51 vs. $36 \%, p=0.004)$ and prophylactic nasogastric tubes (11 vs. $5 \%, p=0.024)$ beyond the first postoperative day was correlated with POI as well as intra- (64 vs. $48 \%, p=0.002)$ and postoperative epidurals (EDA) (43 vs. $32 \%, p=0.028$ ). On POD 3, patients with POI were significantly less ambulant $(4.5 \pm 2.8$ vs. $6.9 \pm 2 \mathrm{~h}, p \leq 0.001)$ and presented with more excess weight $(2.9 \pm 3.9$ vs. $1.6 \pm 2.9, p \leq 0.001)$.

Patients with POI were significantly less compliant to the ERAS pathway (compliance of $>70 \%: 41$ vs. $62 \%, p \leq 0.001$ ).

Multivariate analysis retained the ASA group 3-4 (OR 1.3; $95 \% \mathrm{CI} 1-1.8, p=0.043)$ and duration of surgery of $>3 \mathrm{~h}$ (OR $1.3 ; 95 \%$ CI $1-1.7, p=0.047$ ) as independent risk factors for POI. Minimally invasive surgery (OR 0.6 ; $95 \%$ CI $0.5-0.8$, $p \leq 0.001)$ and overall compliance of $>70 \%$ to the ERAS protocol (OR 0.7; 95\% CI 0.6-1, $p=0.031$ ) represented independent protective factors (Fig. 2).

\section{Outcome}

VAS scores were significantly higher in POI patients on POD 3 for pain $(3 \pm 2.8$ vs. $2.1 \pm 2.3, p=0.003)$ and nausea $(2.1 \pm 3$ vs. $0.8 \pm 1.8, p \leq 0.001)$.

POI was correlated with significantly more major complications (36 vs. $8 \%, p \leq 0.001)$. Striking differences were also 
Fig. 1 Comparison of compliance to pre-, intra- and postoperative ERAS-related items among patients with POI (black bars) and patients without POI (grey bars). Premedication $=$ administration of long-acting sedative medication; $\mathrm{mL} / \mathrm{kg} / \mathrm{h}=$ amount of clear fluid perfusion during the surgical procedure. $P O I$ postoperative ileus, $P O N V$ postoperative nausea and vomiting, $E D A$ epidural analgesia, $P O D$ postoperative day, $N G T$ nasogastric tube. The asterisk indicates statistical significance $(p<0.05)$

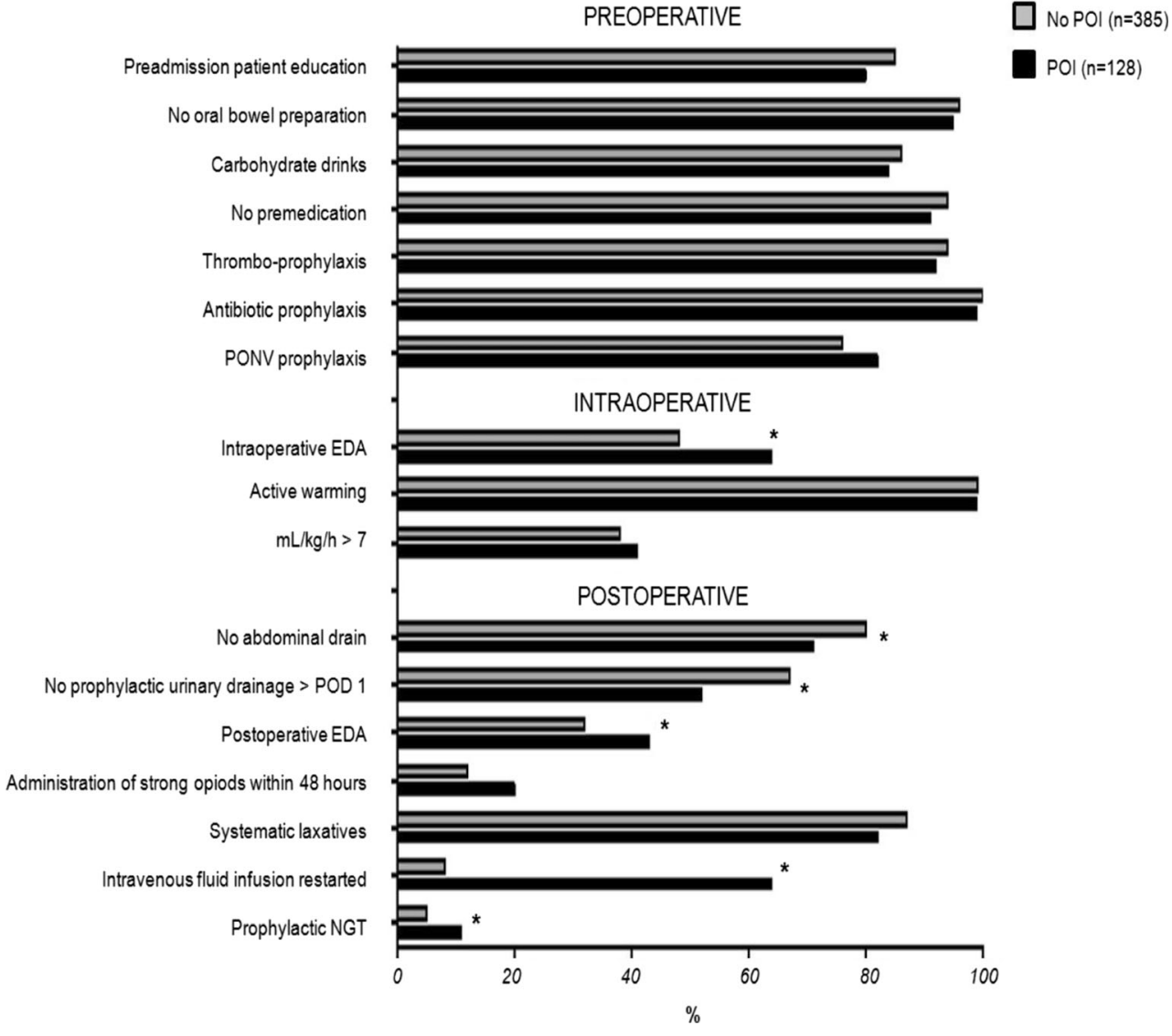

Discussion

Every fourth patient needed postoperative reinsertion of a NGT in the present cohort. This high rate was observed despite treatment of all patients within a standardized enhanced recovery pathway, in which several items aim to prevent this
Fig. 2 Multivariate analysis of univariate risk factors for $\mathrm{POI}$ with $p<0.05$. An odds ratio (OR) of more than one increases the risk of POI. Full lines indicate statistical significance $(p<0.05)$. $E D A$ epidural analgesia, $W H O$ score World Health Organization performance score, $A S A$

American Society of Anesthesiologists

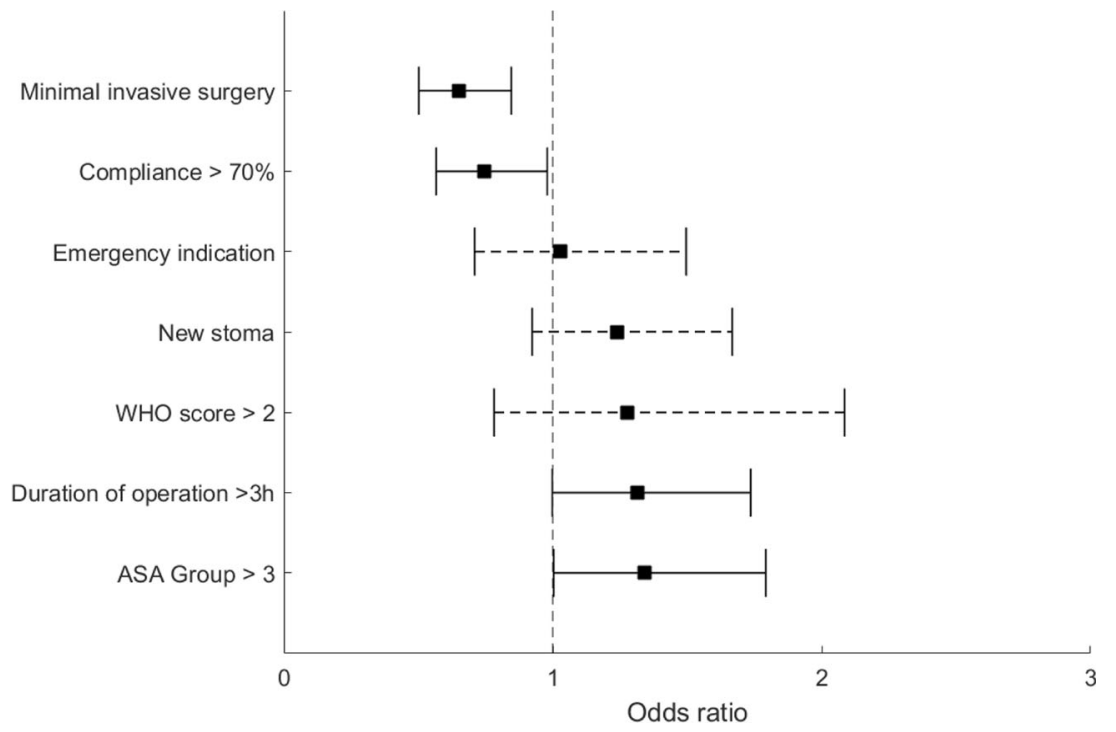




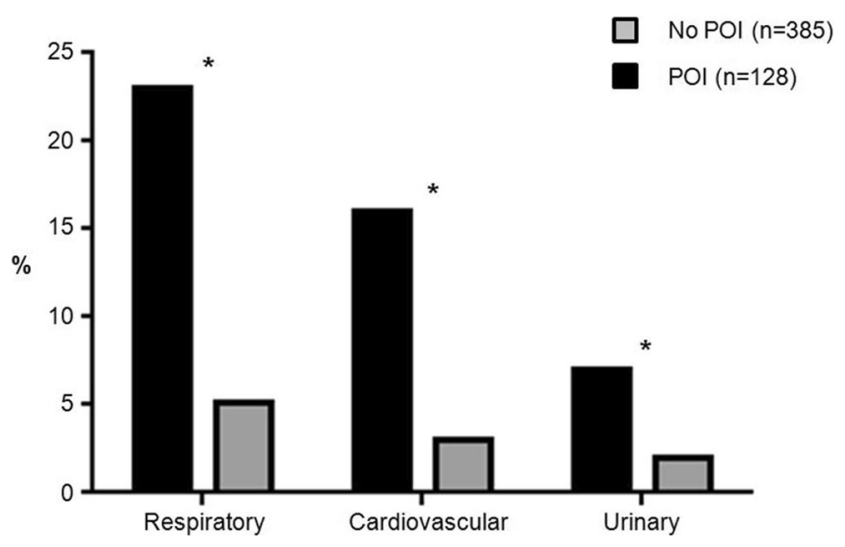

Fig. 3 Comparison of medical complications among patients with POI (black bars) and patients without POI (grey bars). Complications are graded according to the Clavien classification and comprise complications of grade IIIa and higher. POI postoperative ileus. The asterisk indicates statistical significance $(p<0.05)$

displeasing complication. The present study identified long surgical procedure as independent risk factor to contribute to this high ileus rate. Modifiable factors and hence potential targets for improvement were overall compliance to all items of the ERAS protocol and favouring of a minimally invasive approach. Interestingly, different ileus-preventing ERAS items such as laxative administration, avoiding of opioid medication and stringent perioperative fluid management did not differ between the two groups (Fig. 1). This emphasizes the importance to adhere to all items of the protocol, as previously demonstrated by Gustafsson et al. [4].

The disappointingly high rate of POI contrasts with otherwise obvious benefits of ERAS implementation. A recent metaanalysis by Greco et al. showed significantly decreased complication rates and length of stay [6]. A cost-effectiveness analysis by our group showed evident savings [7]. The extension of the protocol to emergent colorectal surgery turned out to be equally feasible, since no significant adverse effect resulted despite higher comorbidity scores and more stressful procedures [8].

Since no consensus has been achieved with regard to the definition of postoperative ileus, a comparison is difficult. Recently, Vather et al. developed a score based on different items: the inability to tolerate oral diet over $24 \mathrm{~h}$, the presence of nausea or vomiting, the absence of flatus over $24 \mathrm{~h}$, the radiologic confirmation occurring on postoperative day 4 and the presence or not of abdominal distension [9]. However, these items are barely objective. Moghadamyeghaneh et al. defined prolonged ileus as no return of bowel function in 7 days, reaching an incidence of $12.7 \%$ in over 27,500 patients of the NSQIP databases [10]. In their cohort, ileus was associated with anastomotic leak and intraabdominal infections, in line with our observations of increased major complication rates. Another analysis of the Premier Perspective database of almost 18,000 patients undergoing colorectal surgery in 2004 in over 500 US hospitals described an ileus rate of $17.4 \%$ when looking for the respective ICD-9-CM codes [11]. The ileus rate in the present cohort was even higher. This might be due to the meticulous prospective assessment of the data. On the other hand, the definition of ileus as postoperative reinsertion of a NGT is objective and easily assessable, as previously described by Wolthuis [12]. In their recently published meta-analysis, the authors propose NGT reinsertion as the most relevant definition of POI after colorectal surgery [12]. A drawback might be an overestimation of ileus rates, assuming that NGTs could have been reinserted for other reasons than POI.

Several pathogenetic factors such as inhibitory neural reflexes, inflammation of the gut wall by direct manipulation and opioids used for postoperative analgesia might contribute to the occurrence of postoperative ileus [11, 13]. The ERAS protocols aim to counteract this inflammatory response by favouring a minimally invasive approach, early food intake and ambulation with systematic administration of laxatives, avoidance of prophylactic nasogastric drainage and opioids and stringent fluid management [3]. Several publications emphasized the importance of compliance to these items in order to achieve best results [4]. Indeed, minimally invasive surgery and its obvious benefits lead to better functional recovery [14]. Laparoscopic resection was an independent predictor of shorter hospital stay compared to open surgery in the LAFA trial, where fast track care was compared to standard care in 400 patients [14]. Significantly decreased ileus rates in the present cohort in minimally invasively operated patients underline these findings. While in the elective setting, colorectal resections were performed whenever possible $(75 \%)$ by laparoscopy, most emergency procedures $(70 \%)$ were performed in an open approach, probably due to lower surgical expertise at night, hemodynamic instability of the patient or bowel dilatation limiting intraabdominal space.

Another point to improve might be more intense preconditioning of severely ill patients by postponing non-emergent surgical indications [15]. Similar to earlier publications [14], the present study revealed more POI with consequent medical complications in patients with ASA grade III or higher (Fig. 2). The POI was associated with respiratory complications, and the high cardiovascular complication rate might reflect cardiac dysrhythmia as a sequel of electrolyte disturbances. It is thus important to emphasize that POI, graded by itself as a minor complication (Clavien grade II) [5], had an alarming potential to induce successive complications in the postoperative course.

The present study demonstrated a significant association of major complications with POI, reflected by increased length of stay. Whether POI did prompt these complications remains speculative. This finding probably rather reflects POI as a consequence of major surgical complications.

In order to lower the incidence of POI, some adaptions within the ERAS protocol need to be discussed. Newer publications advocate the use of preoperative mechanical bowel preparation with oral antibiotics to prevent postoperative 
complications and ileus [16]. The perioperative use of chewing gum is advocated by recent evidence [17]. New anesthesiologic techniques such as the administration of intravenous lidocaine show promising results by reducing postoperative bowel paralysis [18]. A recent publication suggests Alvimopan, a peripherally acting opioid antagonist, as a promising postoperative adjunct in colorectal surgery [19]. In their cohort, Alvimopan administration was associated with faster return of bowel function, lower incidence of POI, shorter hospitalization and cost-effectiveness.

Several limitations of the present study need to be addressed. Even if data collection was performed prospectively, the analysis was conducted retrospectively. Hence, it was difficult to assess whether POI by itself complicated LOS and recovery. Likewise, it was difficult to assess whether POI was promoted by low compliance to the ERAS protocol alone or whether POI did rather impede higher compliance with the postoperative items of the pathway. Some patients were operated for an ileus which did not resolve in the postoperative period, which might overestimate the observed ileus rate. Even by assuming that NGT placement was necessary for POI in most patients, some other indications for NGT placement might overestimate the reported ileus rate. Even if the assessment of POI by NGT reinsertion rates is easily manageable, the call for NGT reinsertion was always upon clinical judgement. A recent Delphi approach delivered an accepted definition of postoperative ileus and defined the criteria for NGT reinsertion [20].This standardization should be helpful to compare the results of future studies on POI.

In conclusion, despite standard care within an enhanced recovery protocol, POI remained a major problem. Adherence to all items of the protocol clearly helped to lower the incidence of POI in the present cohort. Preconditioning of patients and favouring of a minimally invasive surgical approach might help to reduce this frequent complication.

Acknowledgements The authors would like to acknowledge the support provided by all members of the enhanced recovery team in Lausanne, and especially the dedicated nurse V. Addor.

\footnotetext{
Statement of authorship Fabian Grass: design, analysis and interpretation, drafting

Juliette Slieker: design, analysis and interpretation, critical revision

Jonas Jurt: analysis and interpretation, critical revision

Anne Kummer: interpretation, critical revision

Dieter Hahnloser: analysis and interpretation, critical revision

Nicolas Demartines: conception, interpretation, critical revision

Martin Hübner: conception and design, analysis and interpretation, drafting

All authors approved the final version
}

Compliance with ethical standards This study was approved by the Institutional Review Board (Commission cantonale d'éthique de la recherche sur l'être humain (CER-VD)), and informed consent was obtained from every patient.
Conflict of interest The authors declare that they have no conflict of interest.

\section{References}

1. Su'a BU, Hill AG (2015) Perioperative use of chewing gum affects the inflammatory response and reduces postoperative ileus following major colorectal surgery. Evidence-based medicine 20(5):185186

2. Ahmed Ali U, Dunne T, Gurland B, Vogel JD, Kiran RP (2014) Actual versus estimated length of stay after colorectal surgery: which factors influence a deviation? Am J Surg 208(4):663-669

3. Gustafsson UO, Scott MJ, Schwenk W, Demartines N, Roulin D, Francis N, McNaught CE, Macfie J, Liberman AS, Soop M et al (2013) Guidelines for perioperative care in elective colonic surgery: Enhanced Recovery After Surgery (ERAS®) Society recommendations. World J Surg 37(2):259-284

4. Gustafsson UO, Hausel J, Thorell A, Ljungqvist O, Soop M, Nygren J (2011) Enhanced recovery after surgery study Group: adherence to the enhanced recovery after surgery protocol and outcomes after colorectal cancer surgery. Arch Surg 146(5):571-577

5. Dindo D, Demartines N, Clavien PA (2004) Classification of surgical complications: a new proposal with evaluation in a cohort of 6336 patients and results of a survey. Ann Surg 240(2):205-213

6. Greco M, Capretti G, Beretta L, Gemma M, Pecorelli N, Braga M (2014) Enhanced recovery program in colorectal surgery: a metaanalysis of randomized controlled trials. World J Surg 38(6):15311541

7. Roulin D, Donadini A, Gander S, Griesser AC, Blanc C, Hubner M, Schafer M, Demartines N (2013) Cost-effectiveness of the implementation of an enhanced recovery protocol for colorectal surgery. Br J Surg 100(8):1108-1114

8. Roulin D, Blanc C, Muradbegovic M, Hahnloser D, Demartines N, Hubner M (2014) Enhanced recovery pathway for urgent colectomy. World J Surg 38(8):2153-2159

9. Vather R, Josephson R, Jaung R, Kahokehr A, Sammour T, Bissett I (2015) Gastrografin in prolonged postoperative ileus: a doubleblinded randomized controlled trial. Ann Surg 262(1):23-30

10. Moghadamyeghaneh Z, Hwang GS, Hanna MH, Phelan M, Carmichael JC, Mills S, Pigazzi A, Stamos MJ 2015: Risk factors for prolonged ileus following colon surgery. Surgical endoscopy.

11. Iyer S, Saunders WB, Stemkowski S (2009) Economic burden of postoperative ileus associated with colectomy in the United States. Journal of Managed Care Pharmacy : JMCP 15(6):485-494

12. Wolthuis AM, Bislenghi G, Fieuws S, de Buck van Overstraeten A, Boeckxstaens G, D'Hoore A (2016) Incidence of prolonged postoperative ileus after colorectal surgery: a systematic review and meta-analysis. Colorectal disease : the official journal of the Association of Coloproctology of Great Britain and Ireland 18(1): O1-O9

13. Bragg D, El-Sharkawy AM, Psaltis E, Maxwell-Armstrong CA, Lobo DN (2015) Postoperative ileus: recent developments in pathophysiology and management. Clin Nutr 34(3):367-376

14. Vlug MS, Bartels SA, Wind J, Ubbink DT, Hollmann MW, Bemelman WA, Collaborative LSG (2012) Which fast track elements predict early recovery after colon cancer surgery? Colorectal disease: the official journal of the Association of Coloproctology of Great Britain and Ireland 14(8):1001-1008

15. Gillis C, Li C, Lee L, Awasthi R, Augustin B, Gamsa A, Liberman AS, Stein B, Charlebois P, Feldman LS et al (2014) Prehabilitation versus rehabilitation: a randomized control trial in patients undergoing colorectal resection for cancer. Anesthesiology 121(5):937-947 
16. Kiran RP, Murray AC, Chiuzan C, Estrada D, Forde K (2015) Combined preoperative mechanical bowel preparation with oral antibiotics significantly reduces surgical site infection, anastomotic leak, and ileus after colorectal surgery. Ann Surg 262(3):416-425

17. Short V, Herbert G, Perry R, Atkinson C, Ness AR, Penfold C, Thomas S, Andersen HK, Lewis SJ (2015) Chewing gum for postoperative recovery of gastrointestinal function. The Cochrane database of systematic reviews 2:CD006506

18. Sun Y, Li T, Wang N, Yun Y, Gan TJ (2012) Perioperative systemic lidocaine for postoperative analgesia and recovery after abdominal surgery: a meta-analysis of randomized controlled trials. Dis Colon rectum 55(11):1183-1194

19. Adam MA, Lee LM, Kim J, Shenoi M, Mallipeddi M, Aziz H, Stinnett S, Sun Z, Mantyh CR, Thacker JK (2015) Alvimopan provides additional improvement in outcomes and cost savings in enhanced recovery colorectal surgery. Annals of surgery.

20. Gero D, Gie O, Hubner M, Demartines N, Hahnloser D 2016: Postoperative ileus: in search of an international consensus on definition, diagnosis, and treatment. Langenbeck's archives of surgery/ Deutsche Gesellschaft fur Chirurgie. 